Regards sur l'économie allemande

Bulletin économique du CIRAC

115 | 2014

Varia

\title{
Information : les 65 ans de la Bundespressekonferenz
}

Isabelle Bourgeois

\section{OpenEdition}

\section{Journals}

Édition électronique

URL : http://journals.openedition.org/rea/4769

DOI : $10.4000 /$ rea.4769

ISSN : 1965-0787

Éditeur

CIRAC

Édition imprimée

Date de publication : 1 décembre 2014

Pagination : 36-38

ISSN : 1156-8992

Référence électronique

Isabelle Bourgeois, «Information : les 65 ans de la Bundespressekonferenz », Regards sur l'économie allemande [En ligne], 115 | décembre 2014, mis en ligne le 01 décembre 2016, consulté le 15 septembre 2020. URL : http://journals.openedition.org/rea/4769

Ce document a été généré automatiquement le 15 septembre 2020.

(c) CIRAC 


\title{
Information : les 65 ans de la Bundespressekonferenz
}

\author{
Isabelle Bourgeois
}

\section{Concrétisation de la transparence démocratique}

1 En octobre 2014, une institution particulière à la République fédérale a fêté ses 65 ans d' existence: la Bundespressekonferenz (BPK) - littéralement: la conférence de presse fédérale (www.bundespressekonferenz.de). Créée en 1949, cette association (le statut français équivalent serait celui de l'association Loi de 1901) des journalistes politiques allemands et des correspondants étrangers à Berlin garantit à tous ses membres (900) le libre accès à l'information gouvernementale. Chaque lundi, mercredi et vendredi (jours fixes), elle invite le(s) membre(s) du gouvernement de son choix à s'exprimer en public. Traditionnellement, le chancelier ou la chancelière n'est invité(e) qu'une fois par an. Organisation fédérale de l'Allemagne oblige, il existe une Landespressekonferenz dans chacun des 16 Länder, qui procède de même. En Allemagne, les journalistes n'ont donc pas besoin d'accréditation dans l'enceinte d'un ministère ou à la chancellerie ni de faire partie d'un cercle fermé pour être informés sur la politique menée soit par un gouvernement régional, soit par le gouvernement fédéral. Et, surtout, les journalistes étrangers non seulement ne sont pas exclus (ou triés sur le volet), mais ils bénéficient du même droit à l'information que leurs confrères allemands.

\section{Respect du droit à l'information}

2 Ce droit à l'information est en effet inscrit à l'art. 5 de la Loi fondamentale, où il constitue l'autre face, indissociable, de la liberté d'opinion et d'expression. Et le fait qu' il soit institutionnalisé ainsi sous la forme de cette association, c'est-à-dire "par la société civile», atteste que "le droit de la presse fait partie des droits fondamentaux du citoyen» (J. Gauck). Et la Bundespressekonferenz possède un puissant "pouvoir d' organisation » en ce sens que c'est elle qui procède à l'agenda setting, donc détermine les thèmes politiques à débattre dans l'espace public.

Discours de Joachim Gauck pour les 65 ans de la BPK

3 Le 14 octobre 2014, pour célébrer son anniversaire, la BPK avait invité le président de la RFA, Joachim Gauck. Non pas pour le soumettre à 'interrogatoire' (l'exercice est 
redouté des responsables politiques, bien que difficile à esquiver). Mais pour écouter son discours. Et ses critiques, car il n'est pas de discours sur la démocratie en Allemagne sans réflexion sur les faiblesses constatées dans la pratique. Or, à l'ère des TIC, l'information est prise dans l'engrenage de la précipitation, voilà le thème sur lequel intervenait Joachim Gauck (voir www.bundespraesident.de).

\section{La généralisation du turbo journalisme exige un retour au journalisme de qualité}

4 L'accélération des flux de news induite par le saut technologique que nous vivons est "souvent identifiée comme la source de tous nos maux», en Allemagne comme ailleurs. Il mène à un «turbo journalisme » qui sépare les citoyens en insiders et outsiders, ceux qui savent parce qu'ils ont les moyens de s'informer plus amplement, et ceux qui doivent se contenter de 140 signes qu'ils partagent quand les news les font bondir ou qu'ils les «likent». Or la cause des mutations n'est pas cette accélération en elle-même mais " la manière dont nous la gérons ". Il nous faut donc "apprendre à distinguer entre la vitesse de l' information et la précipitation avec laquelle nous émettons un jugement». A fortiori un commentaire. Cela plaide pour "une analyse critique du journalisme par ceux qui le pratiquent ", autrement dit pour un retour au journalisme de qualité. Celui-ci exige du temps, du recul, mais aussi la prise en considération de la demande des lecteurs ou auditeurs qui, pour pouvoir exercer leur rôle de citoyens éclairés, ont besoin d' information. Cela plaide aussi pour la généralisation d'une "éducation aux médias » qui devrait avoir pour règle d'or : "sois sceptique si, à 11 heures 07 , tombe une news à charge contre un homme politique et si, à 11 heures 08 , suit un commentaire ». Enfin, cela rappelle l' importance du respect des grands principes au cœur de la déontologie: véracité, vérification des sources, transparence et sens de la responsabilité. Cela vaut tout particulièrement pour ces " gardiens des écluses " (gate keepers) que sont les membres de la BPK et qui portent de ce fait une lourde responsabilité.

\section{Mode d'emploi du " être-démocratique » (Horst Köhler)}

5 Ce discours n'a rien de convenu. L'information accessible à tous est la base même du fonctionnement de la démocratie allemande : c'est grâce à elle que la RFA a pu s'établir comme une démocratie au sortir des ruines, c'est grâce à elle aussi que, dans cet ensemble polyarchique et hautement complexe qu'est la République fédérale, le peuple peut réellement exercer son pouvoir, puisqu'il participe ainsi aux prises de décision collectives. Plus que jamais, aujourd'hui, le citoyen a un besoin vital d'information - et d'une information de qualité, c'est-à-dire fondée, mûrement réfléchie - pour comprendre le monde dans lequel il vit. Plus encore, soulignait en 2009 Horst Köhler, un prédécesseur de Joachim Gauck, lorsqu'il avait été invité lui aussi à célébrer l' anniversaire de la Bundespressekonferenz (le $60^{\mathrm{e}}$ ): «Le monde dans lequel nous vivons a besoin d'être expliqué pour que nous puissions lui donner forme et consistance. Il n'a jamais été aussi compliqué qu'aujourd'hui ». Lui aussi plaidait pour un journalisme de qualité, seul à même de "donner le mode d'emploi pratique du être-démocratique». Ce journalisme implique une plus grande "tenue». En effet, "le recours au divertissement est important, dans votre métier aussi », disait-il à l'adresse des journalistes, ajoutant : "mais seulement en tant que moyen, pas en tant que succédané d'information ".

\section{Des appels à prévention}

6 A l'ère d'Internet plus que jamais, ce double rappel est utile : le turbo journalisme est la principale cause de la désaffection du lectorat et, partant, de la crise économique (et institutionnelle) de la presse papier. Celle-ci n'offre plus au lecteur ce qu'il cherche: une information factuelle, approfondie, l'amenant à réfléchir, à prendre ses distances 
et à se forger sa propre opinion en connaissance de cause. Mais alors que dans des pays comme la France, cette crise est déjà très profonde, expliquant en partie la montée des populismes, en Allemagne, elle commence seulement à s'esquisser. Les «grands médias" (JT Tagesschau sur la Une publique, radio Deutschlandfunk, quotidiens suprarégionaux comme le F.A.Z.) et même les quotidiens régionaux assurent encore leur fonction d'articulation, d'information, de critique et de contrôle dans la démocratie. Il s 'agit donc plus de prévenir des dérives potentielles avant qu'elles ne gagnent en ampleur et ne soient devenues irréversibles. C'est là le sens des discours d'anniversaire prononcés devant la Bundespressekonferenz.

\section{L'indépendance de la presse est garantie dans la pratique}

Voilà pour les principes. Qu'en est-il de la réalité sur le terrain ? La liberté de la presse est un bien sans prix et les Allemands, ayant fait l'expérience de deux dictatures, lui sont particulièrement attachés. De ce fait, sa pratique fait l'objet d'un suivi régulier qui nourrit de nombreux travaux scientifiques. C'est ainsi par exemple que les éditeurs de presse ont fondé un cercle de mécènes qui soutient non seulement la recherche en la matière, mais également la diffusion des travaux dans un large public, ce qui inclut la pédagogie. Cette Stiftervereinigung der Presse e.V. (autre association de type Loi 1901), a ainsi commandé à l'Institut für Demoskopie d'Allensbach (le plus réputé des instituts de sondages, le plus scientifique aussi), une enquête sur le sujet. Sa conclusion est rassurante : $93 \%$ des journalistes estiment que la liberté de la presse est bien (63\%) ou très bien ( $30 \%)$ garantie en Allemagne. La dernière vague de sondages, qui portait sur leur expérience en matière de tentatives d'influence extérieures, a été réalisée de mars à avril 2014 auprès de 432 journalistes (rédacteurs en chef, responsables des rubriques : politique, économie, informations locales) de plus de 230 quotidiens.

\section{Mais les tentatives de prise d'influence changent de forme}

Dans leur travail, $60 \%$ des sondés n'en affirment pas moins avoir subi des pressions sous différentes formes: entraves lors d'enquêtes, tentatives d'influencer les sujets, pressions économiques... Contrairement à ce qu'on pourrait penser, ces pressions sont particulièrement fortes dans l'information culturelle/musicale et, moins surprenant, sportive. Dans la vague de sondage de l'automne dernier, les taux de réponse se situaient entre respectivement $83 \%$ et $87 \%$. Comme en France, c'est ensuite l' information locale (64\%) qui est la proie de telles tentatives, suivie de l'information politique $(59 \%)$ et économique (54\%). Ceux qui tentent d'influencer les journalistes sont le plus souvent les entreprises (64\%), surtout à l'échelon local (67\%). Les responsables politiques montrent généralement plus de retenue ( $40 \%$ seulement des citations), sauf au niveau local (61\%). Il en va de même des fédérations professionnelles ou d'employeurs ( $41 \%$ ). Quant aux ministères, ils se montrent peu actifs ( $18 \%$ des cas seulement), ce qui est rassurant quant à l'indépendance de la presse par rapport au politique. Certes, $47 \%$ des sondés disent que ces tentatives d'influence ont augmenté dans l'ensemble, mais autant disent ne pas voir de différence. Peut-être l'explication réside-t-elle dans les nouvelles formes que prennent ces tentatives: les cadeaux ou autres prestations en nature sont en recul. Tout à l'inverse d'une pratique qui tend à se généraliser : la réécriture des interviews avant autorisation. Mais la presse l'épingle régulièrement - et publiquement -, la meilleure parade qui soit. Dans l'acception allemande, la démocratie est un organisme vivant, avec ses forces et faiblesses humaines. Elle n'est jamais acquise. Voilà pourquoi, à intervalles réguliers, une réflexion collective s'impose dans la profession. Quelle meilleure occasion que le $65^{\mathrm{e}}$ 
anniversaire d'une institution sans équivalent dans le monde comme cette Bundespressekonferenz?

INDEX

Mots-clés : droit, économie du savoir, médias, nouvelles technologies, presse 